Courrier du Centre international Blaise Pascal

36 | 2014

Varia

\title{
Pascal via Duhem et van Fraassen
}

\section{Vincent Jullien}

\section{OpenEdition}

Journals

Édition électronique

URL : http://journals.openedition.org/ccibp/311

DOI : $10.4000 /$ ccibp. 311

ISSN : 2493-7460

\section{Éditeur}

Centre international Blaise Pascal

\section{Édition imprimée}

Date de publication : 5 février 2014

Pagination : 5-12

ISBN : 978-2-84516-693-6

ISSN : 0249-6674

\section{Référence électronique}

Vincent Jullien, «Pascal via Duhem et van Fraassen », Courrier du Centre international Blaise Pascal [En ligne], 36 | 2014, mis en ligne le 03 décembre 2015, consulté le 01 mai 2019. URL : http:// journals.openedition.org/ccibp/311; DOI : 10.4000/ccibp.311

Ce document a été généré automatiquement le 1 mai 2019.

Centre international Blaise Pascal 


\title{
Pascal via Duhem et van Fraassen
}

\author{
Vincent Jullien
}

\section{La place discrète de Pascal dans l'histoire de la philosophie des sciences}

1 Nous nous intéresserons ici à la physique pascalienne et à l'écho qu'elle a eu dans l'histoire de la discipline. Il est facile de montrer la faible présence de Pascal dans la pensée épistémologique moderne et contemporaine en examinant quelques données puisées dans un échantillon restreint mais significatif de la bibliographie susceptible de présenter ou de commenter l'épistémologie physique pascalienne. Le gros volume dirigé par Pierre Wagner, Les philosophes et la science ${ }^{1}$ est assez complet et on y rencontre presque tout le monde. Pascal n'y a aucune place, ou plutôt la place d'un ciron : une note de fin de texte pour indiquer qu'il condamne l'entreprise cartésienne, en s'appuyant sur le fragment $84^{2}$ : «Descartes. Il faut dire en gros : cela se fait par figure et mouvement etc.» Le contresens habituel, largement démonté par Vincent Carraud est une fois de plus réactivés.

2 Un traitement identique lui est réservé dans l'ouvrage collectif La philosophie des sciences au XXe siècle $e^{4}$. Seule une mention indiquant qu'il a contribué avec Fermat à la mise à jour des « lois du hasard » témoigne de l'œuvre pascalienne.

Parcourons des textes aussi variés que Adieu la raison de Paul Feyerabend ${ }^{5}$, Intuitions de génies d'Arthur Miller ${ }^{6}$, Sciences et religion, de Copernic à Galilée ${ }^{7}$, Esquisse d'une sémiophysique de René Thom ${ }^{8}$, La science et l'hypothèse de Poincaré, Histoire et méthodologie des sciences d'Imre Lakatos ${ }^{9}$, La connaissance objective de Karl Popper ${ }^{10}$, La structure des révolutions scientifiques de T. Kuhn ${ }^{11}$, mais aussi La formation de l'esprit scientifique ou Le nouvel esprit scientifique de Gaston Bachelard ${ }^{12}$ (ce ne sont là, disons-le encore, que des exemples), nous ne trouvons pas trace de l'auteur du Traité de l'équilibre des liqueurs. Chez Bachelard particulièrement, on aurait cru devoir le rencontrer, si l'on songe au programme de constitution d'une « épistémologie non cartésienne ${ }^{13}$ ». 
4 Les prises de positions d'Alexandre Koyré méritent un commentaire spécial dans la mesure où il est un des auteurs les plus autorisés en ce qui concerne la science classique. Elles sont particulièrement sévères au sujet de Pascal physicien puisque Koyré avance trois choses : le verbe pascalien est magique et cette magie est dangereuse car elle nous induit en erreur; les expériences pascaliennes sont sujettes à caution et s'il les a faites, il ne nous les a pas décrites comme elles se déroulèrent; la méthode ou l'épistémologie pascalienne ne méritent pas de commentaire particulier si ce n'est de les reconnaître inspirées par (voire copiées sur) celles de Gassendi notamment ${ }^{14}$. Au total, A. Koyré n'a au mieux - rien à dire sur l'apport épistémologique de Blaise Pascal.

Chez deux auteurs un peu plus anciens, Émile Meyerson, De l'explication dans les sciences ${ }^{15}$ et Ernest Renan, L'avenir de la science ${ }^{16}$, on rencontre des jugements certes " marginaux ", mais intéressants.

Meyerson " range » Pascal dans la lignée d'Aristote contre Bacon, et surtout, contre les positivistes en indiquant que l'esprit scientifique est - chez les premiers - mû par la curiosité, la libido sciendi, et pas par l'instrumentalisation. Il cite la célèbre assertion du Stagirite, «l'homme a naturellement la passion de connaître » et évoque l'existence d'un savoir « dont l'objet ne peut être ni l'agrément ni le besoin », savoir dont il retrouve la trace à la Renaissance même, ce que Montaigne exprime sans équivoque : « Il n'est désir plus naturel que le désir de connaissance...» Pour Pascal, de même et toujours selon Meyerson, il semble aller de soi que « les curieux et les savants... ont pour objet l'esprit » et que, dans les choses spirituelles, règne "la curiosité proprement ». À rebours, Auguste Comte, tout en ne niant pas absolument cet esprit de curiosité scientifique, déclare que ce penchant est « un des moins impérieux de notre nature ${ }^{17}$ ».

Ernest Renan, de son côté, contribue à forger l'image du scepticisme pascalien.

Le grand scepticisme a toujours été peu caractérisé en France ; à commencer par Montaigne et Pascal, nos sceptiques ont été ou des gens d'esprit ou des croyants, deux scepticismes très voisins l'un de l'autre, et qui s'appuient réciproquement ${ }^{18}$.

Et plus loin :

Le scepticisme s'échelonne ainsi aux divers degrés de l'intelligence humaine, alternant avec le dogmatisme selon le développement plus ou moins grand des facultés intellectuelles. Au plus humble degré, est le dogmatisme absolu des ignorants et des simples, qui affirment et croient par nature et n'ont pas aperçu les motifs de douter. Quand l'esprit, longtemps bercé dans cette foi naïve, commence à découvrir qu'il a pu être le jouet de sa croyance, il entre en suspicion, et s'imagine que le plus sûr moyen pour ne pas être trompé, c'est de rejeter toute chose: premier scepticisme qui a aussi sa naïveté (sophistes, Montaigne, etc.). Un savoir plus étendu, prenant la nature humaine par son milieu, sans s'inquiéter des problèmes radicaux, essaie ensuite de fonder sur le bon sens un dogmatisme raisonnable, mais sans profondeur (Socrate, Th. Reid). Plus de vigueur d'esprit montre bientôt le peu de fondement de cette nouvelle tentative; on s'attaque à l'instrument même: de là un grand, terrible, sublime scepticisme Kant, Jouffroy, Pascal). Enfin, la vue complète de l'esprit humain, la considération de l'humanité aspirant au vrai et s'enrichissant indéfiniment par l'élimination de l'erreur, amène le dogmatisme critique, qui ne redoute plus le scepticisme, car il l'a traversé, il sait ce qu'il vaut, et, bien différent du dogmatisme des premiers âges, qui n'avait pas entrevu les motifs du doute, il est assez fort pour vivre face à face avec son ennemi ${ }^{19}$

Remontons encore dans le temps, du côté de l'Encyclopédie. Chez Diderot et d'Alembert, on rencontre deux choses: un jugement quasi psychologique du premier se désolant du 
gâchis d'un génie par l'idéologie janséniste ${ }^{20}$, et une analyse précise du Traité de l'équilibre des liqueurs par le second qui en fixe les apports et les limites ${ }^{21}$.

Le rôle extraordinairement modeste dévolu à Pascal contraste avec le jugement porté par son grand éditeur actuel, Jean Mesnard qui estime que Pascal " pose avec vigueur les règles de la méthode scientifique: solidité des principes, qu'ils soient évidents d'euxmêmes ou conséquences d'autres principes, rigueur des raisonnements ${ }^{22}$ ». Pour le coup, c'est presque trop! Trop vague quant à ce que serait la méthode scientifique et trop inconditionnel et généreux quant à l'apport pascalien. L'hommage est trop formel pour renverser la situation.

\section{Pierre Duhem et Bas van Fraassen, deux grands contrepoints}

Nous allons nous intéresser à deux auteurs majeurs de la pensée épistémologique du XX siècle qui offrent un contrepoint radical à cette mise à l'écart. Duhem et van Fraassen se donnent les moyens d'étayer leur conviction selon laquelle Blaise Pascal est bien la source essentielle d'une saine philosophie de la science.

Pierre Duhem installe Pascal à tous les moments clé de sa conception de la nature, de l'objet et de la structure de la Théorie Physique. Chaque chapitre de son ouvrage éponyme et chaque moment décisif de son argumentation est en quelque sorte mis sous la bannière de Pascal. Dès le début, lorsqu'il s'agit de critiquer la présence des « cosmologies » dans le développement de la science; ensuite pour étayer l'équilibre nécessaire entre le réalisme et le conventionnalisme ; ainsi encore pour analyser les qualités et les styles des esprits scientifiquement créatifs et pour caractériser le statut de la vérité dans les sciences physiques. Enfin, le livre se clôt par un hommage dans lequel Pascal est donné comme la boussole à laquelle devra se fier le scientifique ${ }^{23}$.

Nous verrons plus précisément ce que l'on peut en penser.

Le projet de Bas van Fraassen est de constituer une doctrine de ce qu'il nomme l'empirisme constructif. Dans deux de ses ouvrages, The scientific image (1980) et Laws and symetries (1989), il s'agit de lutter contre l'empirisme logique d'une part, contre le réalisme d'autre part, ou encore contre le dogmatisme et le scepticisme. Insistant sur ce conflit, il trouve la figure inaugurale de Pascal.

Une nouvelle théorie de la connaissance a grandi discrètement au milieu des ruines de l'ancienne [...]. Outre Descartes et Newton, le dix-septième siècle nous a aussi donné Blaise Pascal. Les écrits de Pascal [...] ont donné naissance à un courant qui s'est transformé au cours des trois siècles suivants en un fleuve puissant : celui de la théorie underground de la connaissance et de l'opinion probables [...] (p. 15)

Catherine Chevalley, dans sa présentation de la traduction française de Laws and symetries note que

la conception de la connaissance qu'on trouve chez Pascal est unique au XVII siècle par la manière dont elle s'oppose au projet de fonder les mathématiques et la physique dans une métaphysique (p. 16).

16 Et aussi que

Loi et symétrie propose [...] de revenir à la façon même dont le problème de la connaissance avait été posé au début de la philosophie moderne, et à contrecourant d'elle, par Pascal (p. 23). 
Elle a raison d'ajouter que c'est «l'existence de la tradition pascalienne qui autorise van Fraassen à n'être pas sceptique en ce qui concerne la possibilité de renouveler la conception de la connaissance » (p. 23).

\section{Van Fraassen reconnaît sa dette duhémienne}

Le rapport qui semble se constituer entre Blaise Pascal, Pierre Duhem et Bas van Fraassen est d'ailleurs enregistré et explicite chez celui-ci, qui reconnaît sa proximité avec Duhem. J'en donne quelques indices objectifs.

19 Le premier texte qu'il cite est tiré de Sauvez les phénomènes de Pierre Duhem (p. 68). Ensuite, van Fraassen mentionne la tâche qui fait l'objet de son travail en ces termes : « il faut reposer la question du «rôle et de la structure (Aim and structure) de la science» (p. 27). On reconnaît le titre presque exact du livre de Duhem.

Sa remarque (p. 33) selon laquelle, "les modèles scientifiques peuvent comporter de nombreux éléments de structure qui ne correspondent absolument à aucun élément de réalité, sans pour autant que cela nuise à leur fonction ", est très proche de la thèse duhémienne du modèle mathématique d'une théorie physique.

21 Nous découvrons un indice supplémentaire lorsque, à un moment plutôt central de son argumentation (la présentation de ce qu'il faut comprendre par équivalence entre théories, ou entre modèles), van Fraassen emploie l'exposé (et aussi le vocabulaire) de Pierre Duhem (tiré de Sauver les phénomènes) : « le premier usage explicite connu de l'idée d'équivalence a porté sur deux hypothèses concernant le mouvement du soleil. Les anciens grecs avaient remarqué... Hipparque démontra etc. » (p. 339). C'est une véritable paraphrase des pages 6 et 7 de Sauver les phénomènes ${ }^{24}$.

Nouvelle preuve dans le paragraphe Qu'est-ce que l'interprétation? (p. 345). Van Fraassen commence par rappeler le programme de Duhem concernant la «mise à l'écart de la métaphysique ", estimant par ailleurs que le physicien français ne parvint pas vraiment à le réaliser (van Fraassen tire ce jugement de l'appendice "Une physique de croyant", ajouté par Duhem à son ouvrage).

J'ajouterai enfin l'exposé par van Fraassen des «trois niveaux de la théorie » (p. 345 sq.) : « Nous apercevons trois niveaux : celui de la représentation des phénomènes donnée par la théorie, celui de la théorie en elle-même et celui des interprétations de la théorie " (p. 347). Voilà qui ressemble fort aux niveaux duhémiens qui structurent une théorie physique.

\section{Comment interpréter la mobilisation de Pascal par Duhem et van Fraassen?}

Si Pierre Duhem et Bas van Fraassen se recommandent aussi intensément de Pascal, c'est qu'ils estiment y trouver des appuis à telle ou telle de leurs thèses épistémologiques. Or, justement, le statut des lois de la nature peut constituer un de ces appuis, quoiqu'il n'ait pas tout à fait le même sens chez l'un et chez l'autre. 


\section{Que cherche Pierre Duhem chez Pascal ?}

Il est indéniable que le vocabulaire pascalien laisse peu de place, voire aucune, à la notion de « lois de la nature », pour des raisons sur lesquelles on pourra revenir. Comme le note c. Chevalley,

La singularité de la physique de Pascal au XVII siècle est d'apporter une contribution majeure à la connaissance des phénomènes sans faire le moindre usage de la notion de loi universelle et nécessaire ${ }^{25}$.

Or, un examen du lexique de la Théorie physique montre que Pierre Duhem ne fait pas davantage de place aux « lois de la nature » : on en trouve deux occurrences, en citation d'autres auteurs (p. 256 et 264 ; on rencontre une fois le terme de «loi générale » (à propos des forces créant un mouvement curviligne, p. 381). Si l'on trouve de nombreuses occurrences de «loi physique» (qui est même dans le titre du chapitre 2, V.), c'est systématiquement pour désigner un résultat local et évidemment éphémère (la loi de Mariotte, les lois de Kepler, etc.).

7 En revanche, la notion de "classification naturelle " est extrêmement fréquente (pas moins de 24 occurrences et toujours comme pièce centrale de l'argumentation). On ne peut pas, ici, exposer la richesse de cette expression, qui semble plutôt appartenir aux naturalistes, et que Duhem étend ou importe en physique. Cette "classification naturelle» est l'ordre profond, ultime et réel de la nature, vers lequel tendent progressivement les théories physiques, au fur et à mesure de leur succession conceptuelle et historique. Or, c'est justement cela que Duhem cherche chez Pascal : un va-et-vient entre l'instabilité et la nature provisoire des connaissances naturelles d'une part, et un ordre naturel qui, bien qu'inaccessible, sert d'horizon et d'attirance à l'esprit et l'activité du savant. Duhem paraphrase Pascal en écrivant que

le développement de la physique provoque une lutte continuelle entre la nature qui ne se lasse pas de fournir et la raison qui ne veut pas se lasser de concevoir (p. 29).

\section{Il s'approprie l'héritage pascalien en affirmant :}

Par une intuition où Pascal eût reconnu une de ces raisons du cœur « que la raison ne connaît pas ", [le savant] affirme sa foi en un ordre réel dont ses théories sont une image, de jour en jour plus claire et plus fidèle. Ainsi l'analyse des méthodes par lesquelles s'édifient les théories physiques nous prouve, avec une entière évidence, que ces théories ne sauraient se poser en explication des lois expérimentales; et, d'autre part, un acte de foi que cette analyse est incapable de justifier, comme elle est impuissante à le refréner, nous assure que ces théories ne sont pas un système purement artificiel, mais une classification naturelle. Et l'on peut, ici, appliquer cette profonde pensée de Pascal :

«Nous avons une impuissance de prouver invincible à tout le Dogmatisme; nous avons une idée de la vérité invincible à tout le Pyrrhonisme. » (p. 36)

29 Duhem termine son livre en tissant un nouveau fil qui le relie au savant janséniste :

Chaque fois que l'esprit du physicien est sur le point de verser en quelque excès, l'étude de l'histoire le redresse par une correction appropriée ; pour définir le rôle qu'elle joue à l'égard du physicien l'histoire pourrait emprunter ce mot de Pascal : « S'il se vante, je l'abaisse ; s'il s'abaisse, je le vante ». Elle le maintient ainsi en cet état de parfait équilibre d'où il peut sainement apprécier l'objet et la structure de la théorie physique (p. 411).

Il aurait pu convoquer aussi la Préface sur le traité du vide rédigée en 1651, où l'on apprend que 
les secrets de la nature sont cachés ; quoiqu'elle agisse toujours, on ne découvre pas toujours ses effets : le temps les révèle d'âge en âge, et quoique toujours égale en elle-même, elle n'est pas toujours également connue [...].

31 S'il est ainsi assez aisé de découvrir ce que Duhem cherchait chez Pascal, il est plus délicat de juger de la validité de sa quête. Autrement dit, n'est-ce pas une lecture quelque peu « forcée » de Pascal à laquelle se livre Duhem? De nombreux passages des grands textes épistémologiques de Pascal iraient dans ce sens: Disproportion de l'homme bien sûr, le fragment 429 aussi qui affirme que « la nature [n'offre rien] qui ne soit matière de doute et d'inquiétude ».

Pierre Duhem visait une super « loi de la nature », avec sa classification naturelle (ou un ensemble de lois qui la constituerait); Pascal, c'est le moins qu'on puisse dire, ne s'engage pas nettement dans cette voie.

Il va aussi sans dire que Duhem cherchait et trouvait, chez Pascal, une tradition critique du cartésianisme. Et aussi qu'il cherchait et trouvait, en Pascal, un savant profondément croyant qui dissociait cette croyance essentielle de ses activités et de ses réflexions scientifiques, qui ne faisait pas dépendre celles-ci de celles-là.

\section{Que cherche van Fraassen chez Pascal?}

Encore plus radicalement que Duhem, van Fraassen cherche une source classique pour son projet de disqualifier la notion de « lois de la nature ». van Fraassen est un théoricien de l'« empirisme constructif », opposé aux diverses formes de réalisme en vogue dans les années 70-80. Son objectif est de «renoncer au concept traditionnel de lois de la Nature au profit de celui de symétries » (p. 8), ce qui conduirait à une " conception entièrement nouvelle » de la connaissance (VII, 257). Les mots qu'il choisit désignent explicitement Pascal :

Il faudra rechercher une théorie de la connaissance et de l'opinion qui ne soit acculée, ni au "désespoir sceptique», ou au relativisme, ni au "réalisme métaphysique » (VII, 256, p. 11).

Comment ne pas être dogmatique, comment ne pas être sceptique? (12)

Je n'expose pas non plus ici les grandes lignes de la thèse de van Fraassen. Retenons seulement que le concept de « lois de la nature » est jugé inutile et néfaste :

Inutile parce que ses fonctions traditionnelles sont aussi bien remplies par les concepts de symétrie et d'invariance, et susceptible de bloquer la réflexion philosophique sur la science parce qu'il fait passer " par la bande », l'hypothèse que la science reflète la structure de la réalité26 (p. 12).

Pour disqualifier la « loi de la nature ", il convient de montrer que la science n'est ni une explication (bien sûr), ni même une représentation, mais une interprétation, du début à la fin du processus. (p. 19). Nous posons des modèles (par exemple les tenseurs et les groupes) qui ont leurs lois ; celles-ci ne sont pas des lois de la Nature. Si cette conception est certainement duhémienne, il n'est pas sûr que l'on rencontre véritablement cette démarche chez Pascal, notamment la notion de modèle disjoint des phénomènes ${ }^{27}$.

Pour la disqualifier, van Fraassen affecte la notion de «loi de la nature » de son sens le plus fort possible: "La loi de la nature est à la fois un don de Dieu et une base axiomatique de la physique» (p. 13). Elle doit être théologique, donc réaliste et formelle. Or, l'histoire de l'idée de «loi de la nature » a montré qu'il y avait d'autres possibilités, moins exigeantes: des lois générales mais non nécessaires ${ }^{28}$. Ce dispositif consiste à 
caricaturer l'adversaire pour le combattre plus facilement. Si telles sont les «lois de la nature ", alors il est vrai qu'elles ne se rencontrent pas chez Pascal où la physique n'est ni déduite de, ni même garantie par Dieu. De plus elle n'est - en principe - pas d'abord axiomatique, mais expérimentale; ce dernier point demandant à être vu de plus près si l'on considère le style synthétique du Traité de l'équilibre des liqueurs.

On peut estimer que van Fraassen demande "trop" à un énoncé pour qu'il puisse acquérir le statut de «loi »: «Dans la pensée philosophique, les lois de la nature ont été caractérisées essentiellement par la généralité et par la nécessité » (p. 414). La généralité, certes, la nécessité, pas toujours. La difficulté n'échappe d'ailleurs pas à van Fraassen qui mentionne une telle distinction explicitée par Hermann Weyl (p. 415) qui assume une doctrine que l'on peut faire remonter à Leibniz, selon laquelle, les lois peuvent être à la fois contingentes et universelles (valables pour tous les mondes) ${ }^{29}$. Il conclut son chapitre (et son livre) en écrivant: «Si nous avons trouvé une notion significative de généralité (pour les lois), nous n'en avons trouvé aucune de nécessité » (417).

Il décide alors de placer Pascal à la source de cette façon de voir :

Le concept de loi de la Nature est une rémanence d'un état de la science qui a été définitivement dépassé (p. 16).

Pascal et ses successeurs ont ouvert pour nous une voie d'une conception entièrement nouvelle de tous les problèmes de la théorie de la connaissance (...). Nous devons penser en termes de probabilité (p. 15).

Van Fraassen choisit ses termes pour valider cette proximité pascalienne ; ainsi lorsqu'il note que « nous ne sommes pas vraiment adéquats au monde; au lieu d'être des miroirs du monde, nous sommes plutôt empêtrés dans les fils de la tapisserie que nous tissons nous-mêmes » (p.18), c'est indiscutablement un thème pascalien, exposé par exemple dans le fragment Disproportion de l'homme, où il est établi que nous ne sommes pas adéquats au monde. Cette idée est réaffirmée par van Fraassen lorsqu'il défend que " nous sommes toujours dans un rapport ambigu avec le monde » (p. 19).

41 Contre ce concept insatisfaisant de Loi, on connait la solution qui a les faveurs de van Fraassen ; il s'agit d'adosser les théories, non plus à des lois de la nature, mais aux symétries et à leurs propriétés mathématiques (p. 41) ${ }^{30}$. Il expose en détail une conquête, un résultat physique obtenu grâce au principe de symétrie : c'est le cas de la composition selon la règle du parallélogramme (il cite Stevin, Lamy, Varignon et Newton); un monde qui ne la respecterait pas est difficilement imaginable.

42 L'argument le plus clair de van Fraassen contre les lois est sans doute celui-ci : les lois sont revendiquées parce qu'elles expliquent, c'est-à-dire parce qu'elles répondent au pourquoi. Or, selon lui, les questions pourquoi sont des questions comme les autres, et reçoivent comme réponse simplement des informations scientifiques plus complètes, mais rien d'ultime. Les lois seraient les limites de régressions infinies, ce qui n'est en général pas atteignable. Il est par ailleurs juste qu'une telle exigence de loi ne se rencontre pas chez Pascal.

43 «En étudiant la science comme si elle était une recherche des lois de la nature, on supposait que la structure de la science doit être comprise comme le reflet de la structure de la nature » (p. 355). Van Fraassen argumente contre cette illusion, ce préjugé dont il convient de douter. Il soutient que ce doute serait aussi pascalien. Ce ne serait peut-être pas faux si l'on pouvait exhiber une structure de la science pascalienne. Ses arguments - modernes - sont forts ${ }^{31}$ : les symétries, auxquelles nous croyons spontanément, nous les introduisons dans nos modèles alors que (dans des cas 
importants), rien ne les garantit (ni même ne les présente) dans la nature : l'aiguille de boussole de Mach tourne toujours du même côté (asymétrie) alors qu'un premier modèle géométrique est symétrique (dans ce cas il y a des variables asymétriques cachées : par exemple le champ terrestre) ; avec les photons de Bell qui se répartissent « sans raison " selon les fentes parallèles, on n'a pas de variable cachée et on produit un modèle statistique symétrique sans que l'on sache ce qu'il en est de cette symétrie «dans la nature "; avec le temps, les équations (les modèles) sont symétriques et cependant, le glaçon ne se « reforme pas ». Je ne discute pas ici de la justesse de ce point de vue, mais de la légitimité de se prévaloir de Pascal pour le soutenir. Des objections peuvent être faites à une telle captatio : 1. la disqualification des lois de la nature (qui réclamaient des causes ultimes explicitées) va de pair avec une valorisation plus grande encore des modèles et des structures mathématiques à travers lesquelles on approcherait les phénomènes. Rien de tel chez Pascal qui tourne le dos plus nettement encore à la physique mathématique qu'à l'idée de loi de la nature. Il est difficile de faire de Pascal l'inventeur, ou même le précurseur de la notion de "symétrie» destinée à remplacer les défuntes «lois". Van Fraassen a bien entendu repéré en Leibniz l'auteur classique qui conçoit et fait un usage décisif de la symétrie (des effets aux causes notamment). Soit, mais il est impossible de mésestimer l'importance majeure du concept de «loi de la nature» dans la philosophie naturelle de Leibniz (et personne n'y songe). Autrement dit, et si l'on suit van Fraassen, celui qui assume l'abandon des « lois » (Pascal) n'est pas le même que celui qui inaugure ou invente le concept qui permet cet abandon (Leibniz); ce dernier, loin d'user du nouvel outil (la symétrie) à ce à quoi il est destiné (l'abandon ou le dépassement des lois), renforce et consolide ce qu'il devrait contribuer à désarticuler.

2. L'originalité radicale de Pascal, pour ce qui concerne le statut de certitude des propositions et des théories physiques, est discutable: Gassendi au premier chef, mais encore Mersenne ou Roberval par exemple, sont les tenants d'une physique " probable ${ }^{32}$ ». Reconnaître ceci risque de banaliser quelque peu la manière pascalienne, d'affaiblir le scénario imaginé par van Fraassen de «l'épistémologie cachée » qu'il parvient enfin à dévoiler. Porter l'attention qu'ils méritent à ces auteurs (comme l'a fait Robert Lenoble ${ }^{33}$ ) conduit immanquablement à reconnaître l'existence et l'influence d'un courant épistémologique plus ou moins continu, avec ses forces et ses faiblesses, mais qui ne peut être une « révélation » méthodologique éblouissante ${ }^{34}$.

La lecture du statut de l'expérience chez Pascal, est plutôt obscure chez van Fraassen : "On notera que l'expérimentation cesse d'avoir un statut rigide (point de départ d'une induction ou point d'arrivée d'une vérification)»(p.33). C'est assez difficilement compatible avec les Expériences nouvelles touchant le vide notamment. Robert Lenoble était plus convaincant lorsqu'il notait: «Avec les expériences, dira Pascal, vont multiplier les principes de notre physique, car la vérité de la nature réside dans ces expériences ${ }^{35} \%$. C'est que, pour van Fraassen, il faut sans doute que les expériences pascaliennes ne puissent vérifier ce qui pourrait être quelque chose comme une loi de la nature.

3. L'objection la plus sérieuse contre la légitimité de se revendiquer si nettement de Pascal sur le statut des vérités générales en physique peut être tirée de l'examen du texte principal de Pascal à ce sujet, c'est-à-dire le Traité de l'équilibre des liqueurs (écrit entre 1650 et 1655). Ce traité est tout entier organisé autour d'une proposition générale qui justifie la presse hydraulique, et à partir de laquelle s'organise toute l'argumentation :

On doit y admirer qu'il se rencontre en cette machine nouvelle cet ordre constant

qui se trouve en toutes les anciennes, savoir le levier, le tour, la vis sans fin etc. qui 
est que le chemin est augmenté en même proportion que la force [...] de sorte que le chemin est au chemin comme la force à la force. Ce que l'on peut prendre même pour la vraie cause de cet effet, étant clair que c'est la même chose de faire faire un pouce de chemin à cent livres d'eau que de faire faire cent pouces de chemin à un litre d'eau ${ }^{36}$. remarque s'impose quant à leur recrue elle-même. Ils lui confient une mission centrale : dessiner, dans la physique classique naissante, un autre chemin que celui du déterminisme, $d u$ réalisme, $d u$ dogmatisme de type cartésien. Outre que ces caractérisations de l'épistémologie cartésienne sont discutables, il n'est pas si sûr que l'anticartésianisme de Pascal soit aussi univoque que l'a estimé une tradition dominante ${ }^{40}$, et surtout, il n'est pas sûr que Pascal puisse véritablement être compté au plein sens du terme comme un "physicien du XVII ${ }^{~}$ ». En effet, sur les grands thèmes de la physique classique naissante, Pascal n'a rien dit : sur les lois du mouvement, sur la mécanique, sur la gravité, sur l'astronomie ou sur l'optique, on serait bien en peine de décrire une épistémologie pascalienne. Si on suit A. Charrak, «au XVII ${ }^{\mathrm{e}}$ et dans de nombreux textes des Lumières, les lois de la nature sont dans les faits synonymes des lois du mouvement, c'est-à-dire des règles de la mécanique ou de la dynamique ${ }^{41} »$. On doit alors en conclure que Pascal est « hors sujet » si celui-ci concerne l'examen des lois de la nature.

De façon générale, Pascal ne s'occupe pas de physique mathématique et, toujours selon Charrak, Pascal anticipe l'idée que se fera Buffon selon laquelle les sciences mathématicomécaniques «trahissent le penchant qu'ont les hommes à vouloir trouver de la ressemblance dans les objets les plus différents, de la régularité où il ne règne que la variété, et de l'ordre dans les choses qu'ils n'aperçoivent que confusément ${ }^{42}$ ». 


\section{NOTES}

1. Wagner Pierre, Les philosophes et la science, Paris, Gallimard, 2005.

2. La numérotation des fragments pascaliens est ici celle de Louis Lafuma.

3. Carraud Vincent, Pascal et la philosophie, P.U.F., 1992, chap. « Descartes », p. 220-280.

4. Barberousse, Kistler, Ludwig, La philosophie des sciences au XX ${ }^{e}$ siècle, Champs, Flammarion, 2000.

5. Feyerabend Paul, Farewell to Reason, 1987 ; Adieu la raison, trad. française par Baudouin Jurdant, Seuil, 1989.

6. Miller Arthur, lnsights of genius, Springer-Verlag, 1996, trad. française Marcel Filoche, Intuitions de génie, Flammarion, 2000.

7. Collection École de Rome, 1999.

8. Thom René, Esquisse d'une sémiophysique, Inter éditions, Paris, 1988.

9. The Methodolbgy of scientific Research Programmes, trad. française Catherine Malamoud et JeanFabien Spitz, P.U.F., 1994. On y trouve une note sur Pascal qui renvoie à une citation de Popkin.

10. Popper Karl, La connaissance objective, Flammarion, 1998, traduit de Objective Knoweldge. An Evolutionnary Approach, Oxford University Press, 1979.

11. Kuhn Thomas, The Structure of scientific revolutions, 1962 (rev. 1970), trad. française, Paris, Flammarion, 1972.

12. Bachelard Gaston, La formation de l'esprit scientifique, Paris, 1938, ou Le nouvel esprit scientifique, Paris, 1934.

13. Après tout, Bachelard n'a sans doute pas eu tort en ne trouvant pas, chez Pascal, d'arguments consistants pour une épistémologie non cartésienne. Quelle qu'en soit la raison, il est absent.

14. On lira le chapitre "Pascal savant» (1954), republié dans Études d'histoire de la pensée scientifique, Tel, Gallimard, 1973, p. 362-389.

15. Meyerson Émile, De l'explication dans les sciences, Paris, Payot, 1921, réédition Corpus des œuvres philosophiques en langue française, Paris, Fayard, 1995.

16. Renan Ernest, L'avenir de la science, Paris, 1890.

17. Les citations sont tirées de De l'explication dans les sciences, 1921, réed. Corpus des œuvres philosophiques en langue française, Paris, Fayard, p. 56-57.

18. L'avenir de la science, p. 1076

19. id., p. 1086.

20. Diderot Denis, Euvres complètes, Garnier, 1875, Pensée philosophique XIV. « Pascal avait de la droiture ; mais il était peureux et crédule. Élégant écrivain, et raisonneur profond, il eût sans doute éclairé l'univers, si la Providence ne l'eût abandonné à des gens qui sacrifièrent ses talents à leurs haines. Qu'il serait à souhaiter qu'il eût laissé aux théologiens de son temps le soin de vider leurs querelles; qu'il se fût livré à la recherche de la vérité, sans réserve et sans crainte d'offenser Dieu, en se servant de tout l'esprit qu'il en avait reçu, et surtout qu'il eût refusé pour maîtres des hommes qui n'étaient pas dignes d'être ses disciples! On pourrait bien lui appliquer ce que l'ingénieux La Mothe disait de La Fontaine. Qu'il fut assez bête pour croire qu'Arnaud, de Sacy et Nicole valaient mieux que lui. »

Pensée XXXI, 4. "C'est l'explication de ceux qui ne veulent pas voir dans Socrate un halluciné comme Pascal. »Ce n'est pas celle de Lélut qui, dans le Démon de Socrate et dans L'amulette de Pascal, croit à un état maladif du cerveau chez ces deux célèbres visionnaires (vol. 2, p. 17).

"Qui est-ce qui ne connaît pas le petit Traité de l'équilibre des liqueurs de Pascal, et la profonde Hydrodynamique de d'Alembert?» (Plan d'une université pour le gouvernement de Russie, p. 339). 
21. Les articles de l'Encyclopédie méthodique mathématique, écrits par d'Alembert (Fluide, hydraulique, Hydrostatique et Hydrodynamique) font une large place au Traité de l'équilibre des liqueurs

\section{Dict. science classique, p. 381.}

23. Page 29 : «Le développement de la physique provoque une lutte continuelle entre la nature qui ne se lasse pas de fournir et la raison qui ne veut pas se lasser de concevoir " (Indiscutable paraphrase de Pascal).

Page 36 : «Par une intuition où Pascal eût reconnu une de ces raisons du cœur « que la raison ne connaît pas ", il affirme sa foi en un ordre réel dont ses théories sont une image, de jour en jour plus claire et plus fidèle. Ainsi l'analyse des méthodes par lesquelles s'édifient les théories physiques nous prouve, avec une entière évidence, que ces théories ne sauraient se poser en explication des lois expérimentales; et, d'autres part, un acte de foi que cette analyse est incapable de justifier, comme elle est impuissante à le refréner, nous assure que ces théories ne sont pas un système purement artificiel, mais une classification naturelle. Et l'on peut, ici, appliquer cette profonde pensée de Pascal : « Nous avons une impuissance de prouver invincible à tout le Dogmatisme ; nous avons une idée de la vérité invincible à tout le Pyrrhonisme. »

Page 80 : «La théorie physique, telle que nous l'avons conçue, ne sera donc pas acceptée d'emblée comme la forme véritable sous laquelle la nature doit être représentée, sinon par les esprits abstraits. Pascal n'en omet pas la remarque en ce fragment $\left(^{*}\right)$ où il caractérise si fortement les deux sortes d'esprits que nous venons de distinguer. »

Page 127: "Le contraste des deux formes intellectuelles si nettement définies par Pascal se marque avec une extraordinaire vigueur dans la discussion célèbre $\left(^{*}\right)$ qui mit aux prises Gassendi et Descartes. »

Page 154 (fin de la première partie) : «La raison n'a donc point d'argument logique pour arrêter une théorie physique qui voudrait briser les chaînes de la rigueur logique; mais la «nature soutient la raison impuissante et l'empêche d'extravaguer jusqu'à ce point $\left(^{*}\right)$ ».

Page 271 : «L'homme peut jurer de dire la vérité ; mais il n'est pas en son pouvoir de dire toute la vérité, de ne dire rien que la vérité. »

«La vérité $\left(^{*}\right)$ est une pointe si subtile que nos instruments sont trop émoussés pour y toucher exactement. S'ils y arrivent, ils en écachent la pointe, et appuient tout autour, plus sur le faux que sur le vrai. »* (PASCAL, Pensées, édition Ravet, art. III. $\mathrm{n}^{\circ}$ 3.)

Page 306 : «Les discussions que nous avons développées ont établi, et de reste, cette vérité : La méthode inductive, dont on recommande l'usage au physicien, lui est aussi impraticable que l'est, au mathématicien, cette méthode déductive parfaite, qui consisterait à tout définir et à tout démontrer, cette méthode à la recherche de laquelle certains géomètres semblent s'acharner, bien que Pascal en ait fait, dès longtemps, bonne et rigoureuse justice. Il est donc bien clair que ceux qui prétendent dérouler, selon cette méthode, la suite des principes de la Physique en donneront forcément un exposé qui sera fautif en quelque point. »

Page 411 (fin du livre) : "Chaque fois que l'esprit du physicien est sur le point de verser en quelque excès, l'étude de l'histoire le redresse par une correction appropriée ; pour définir le rôle qu'elle joue à l'égard du physicien l'histoire pourrait emprunter ce mot de Pascal (*): "S'il se vante, je l'abaisse ; s'il s'abaisse, je le vante." Elle le maintient ainsi en cet état de parfait équilibre d'où il peut sainement apprécier l'objet et la structure de la théorie physique.» (* PASCAL, Pensées, édition Ravet, art. 8.)

24. Dans l'édition Vrin, Mathesis, 1994.

25. Chevalley Catherine, Introduction à Lois et symétrie, Mathesis, Paris, Vrin, 1994, p. 59.

26. Je remarque que la première critique ne fait qu'enregistrer une évolution $d u$ «nom », du « mot » et la seconde suppose le problème résolu : « lois de la nature » est néfaste car il n’y a pas de « lois de la nature ». 
27. En outre, c'est une thèse philosophique forte qui autoriserait à soutenir que les «lois des modèles » ne peuvent être considérées comme des « lois de la nature ».

28. Cf. Charrak, Contingence et nécessité des lois de la nature au XVII ${ }^{e}$ siècle, Paris, Vrin, 2006.

29. On doit atténuer les présentations fréquentes qui isolent exagérément Descartes sur ce point. On lit en effet, dans le Discours de la méthode, cinquième partie, AT VI, 43, Alquié I, 615, la thèse suivante: «De plus je fis voir quelles étaient les lois de la nature; et sans appuyer mes raisons sur aucun autre principe que sur les perfections infinies de Dieu (ici l'immutabilité), je tâchai à démontrer toutes celles dont on eût pu avoir quelque doute et à faire voir qu'elles sont telles, qu'encore que Dieu aurait créé plusieurs mondes, il n'y en saurait avoir aucun où elles manquassent d'être observées. »

30. La symétrie («les problèmes de structure semblable doivent admettre des solutions semblables ", p. 41 et développé p. 355 sq.) se substitue à l'universalité. Les arguments de symétrie sont des recherches d'invariants. Il faut à van Fraassen contester l'idée que toute asymétrie dans la nature ne peut venir que d'une asymétrie antérieure.

31. Voir p. 365 sq.

32. Voir par exemple, Jullien Vincent, «Gassendi, Roberval à l'académie Mersenne. Lieux et occasions de contact entre ces deux auteurs ", XXII siècle, $\mathrm{n}^{\circ}$ 233, octobre 2006, p. 601-613.

33. Lenoble Robert, manuscrits écrits avant 1959 et réunis par Joseph Beaude sous le titre Histoire de l'idée de nature, Paris, Albin Michel, 1969.

34. L'ampleur de cette rupture pascalienne, de la nouveauté de cette voie, laisse un peu dubitatif :

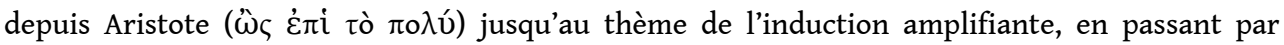
l'équivalence des hypothèses, il est au fond assez commun de penser « les choses qui changent " comme raisonnablement sues, attendues, connues. Ce qui est nouveau (et c'est exactement làdessus que Pascal fait porter le côté saisissant, c'est que la probabilité œuvre aussi en géométrie, la Géométrie du hasard) ; mais le fait que les théories physiques (locales) soient probables ne me semble pas si remarquable.

35. Lenoble Robert, op. cit., p. 313.

36. Traité de l'équilibre des liqueurs, ch. II, éd. Lafuma, p. 237, OC II, éd. J. Mesnard, p. 1045). Ceci renvoie au principe du travail chez Descartes : « c'est la même chose de lever deux cent livres à la hauteur d'un pied et d'enlever cent à la hauteur de deux pieds ».

37. Lettre à Huygens du 5 octobre 1637, AT I, p. 431-448, repris le 13 juillet 1638 à Mersenne, AT III, p. 222-248, puis édité comme Traité de mécanique par Poisson en 1668.

38. Cette dernière affirmation est de Descartes (AT II, 228). Voir Fichant Michel, La notion de force dans la physique de Descartes, in « Descartes et le Rationalisme », 1996, p. 66.

39. Ibid ., p. 69. On pourrait certes dire qu'il s'agit là d'une « loi des machines » et justement pas d'une «loi de la nature»; ce serait instituer une coupure des unes à l'autre, pour le coup artificielle (les «machines simples sont souvent "dans la nature »). C'est peut-être vrai pour Descartes, cf. Fichant p. 68-69.

40. Voir sur ce point les thèses de Vincent Carraud, op. cit., chap.« Descartes ».

41. Op. cit. p. 60.

42. Buffon, De la manière d'étudier l'histoire naturelle, cité in Charrak, p. 55. 
INDEX

Mots-clés : Pascal, Duhem (Pierre), Frassen (van)

\section{AUTEUR}

VINCENT JULLIEN

Centre Atlantique de Philosophie, Université de Nantes 